\title{
GENERALIZED CONVEX FUNCTION AND ASSOCIATED PETROVIĆ'S INEQUALITY
}

\author{
A. UR. REHMAN ${ }^{1}$, G. FARID ${ }^{1}$ AND VISHNU NARAYAN MISHRA $^{2,3, *}$ \\ ${ }^{1}$ COMSATS University Islamabad, Attock Campus, Kamra Road, Attock 43600, Pakistan \\ ${ }^{2}$ Department of Mathematics, Indira Gandhi National Tribal University, Lalpur, Amarkantak, Anuppur, \\ Madhya Pradesh 484 887, India \\ ${ }^{3}$ L. 1627 Awadh Puri Colony Beniganj, Phase - III, Opposite - Industrial Training Institute (I.T.I.), \\ Ayodhya 224 001, Uttar Pradesh, India \\ *Corresponding author: vishnunarayanmishra@gmail.com
}

\begin{abstract}
In this paper, Petrović's inequality is generalized for $h$-convex functions, when $h$ is supermultiplicative function. It is noted that the case for $h$-convex functions does not lead the particular cases for $P$-function, Godunova-Levin functions, $s$-Godunova-Levin functions and $s$-convex functions due to the conditions imposed on $h$. To cover the case, when $h$ is submultiplicative, Petrović's inequality is generalized for $h$-concave functions.
\end{abstract}

\section{INTRODUCTION}

Let $[c, d]$ be an interval containing $(0,1)$ and $h:[c, d] \rightarrow \mathbb{R}$ be a non-negative function. A function $f:[a, b] \rightarrow \mathbb{R}$ is said to be an $h$-convex, if $f$ is non-negative and for all $x, y \in[a, b], \alpha \in(0,1)$, one has

$$
f(\alpha x+(1-\alpha) y) \leq h(\alpha) f(x)+h(1-\alpha) f(y) .
$$

Received 2018-09-12; accepted 2018-11-09; published 2019-01-04.

2010 Mathematics Subject Classification. 52A41.

Key words and phrases. Petrović's inequality; convex functions; $h$-convex functions; concave functions; $h$-concave functions.

(C)2019 Authors retain the copyrights of their papers, and all open access articles are distributed under the terms of the Creative Commons Attribution License. 
If above inequality is reversed, then $f$ is said to be $h$-concave.

The $h$-convex function was introduced by S. Varošanec in [1]. The important thing about these function is that it generalized many other generalization of convex function like $s$-convex functions, Godunova-Levin functions, $s$-Godunova-Levin functions and $P$-functions given in [1-3].

Remark 1.1. Particular value of $h$ in inequality (1.1) gives us the following results:

i. $h(\alpha)=\alpha$ gives the convex functions.

ii. $h(\alpha)=1$ gives the $P$-functions.

iii. $h(\alpha)=\alpha^{s}$ and $\alpha \in(0,1)$ gives the $s$-convex functions of second sense.

iv. $h(\alpha)=\frac{1}{\alpha}$ and $\alpha \in(0,1)$ gives the Godunova-Levin functions.

v. $h(\alpha)=\frac{1}{\alpha^{s}}$ and $\alpha \in(0,1)$ gives the $s$-Godunova-Levin functions of second sense.

In case of $h$-concavity, following results are valid:

vi. $h(\alpha)=1$ gives the reverse $P$-functions.

vii. $h(\alpha)=\alpha^{s}$ and $\alpha \in(0,1)$ gives the $s$-concave functions of second sense.

viii. $h(\alpha)=\frac{1}{\alpha}$ gives the reverse Godunova-Levin functions.

ix. $h(\alpha)=\frac{1}{\alpha^{s}}$ gives the reverse $s-$ Godunova-Levin functions of second sense.

In [6] (also see [7, p. 154]), M. Petrović proved the following result, which is known as Petrović's inequality in the literature.

Theorem 1.1. Suppose that $\left(x_{1}, \ldots, x_{n}\right)$ and $\left(p_{1}, \ldots, p_{n}\right)$ be non-negative $n$-tuples such that $\sum_{k=1}^{n} p_{k} x_{k} \geq x_{i}$ for $i=1, \ldots, n$ and $\sum_{k=1}^{n} p_{k} x_{k} \in[0, a]$. If $f$ is a convex function on $[0, a]$, then the inequality

$$
\sum_{k=1}^{n} p_{k} f\left(x_{k}\right) \leq f\left(\sum_{k=1}^{n} p_{k} x_{k}\right)+\left(\sum_{k=1}^{n} p_{k}-1\right) f(0)
$$

is valid.

In recent years, $h$-Convex functions are considered in literature by many researchers and mathematicians, for example, see $[1,3,5,8,9]$ and references there in. Many authors worked on Petrović's inequality by giving results related to it, for example see $[6,10-12]$ and it has been generalized for $m$-convex functions by $\mathrm{M}$. Bakula et.al. in [13]. In [14], Petrović's inequality was generalized on coordinates by using the definition of convex functions on coordinates.

In this paper, Petrović's inequality is generalized for $h$-convex functions, in the case, when $h$ is supermultiplicative function. In case, when $h$ is submultiplicative, Petrović's inequality is generalized for $h$-concave functions. Also the results has been generalized on coordinates in the plane. 


\section{Generalized Petrović's inequality for h-COnvex function}

A function $h:[c, d] \rightarrow \mathbb{R}$ is said to be a submultiplicative function if

$$
h(x y) \leq h(x) h(y)
$$

for all $x, y \in[c, d]$. If the above inequality is reversed, then $h$ is said to be supermultiplicative function. If equality holds in the above inequality, then $h$ is said to be multiplicative function.

Here we state important lemma, which is very helpful in proving Petrović's inequality for $h$-convex functions. This lemma is generalization of result given in [7, Page 152].

Lemma 2.1. Suppose that $\left(x_{1}, \ldots, x_{n}\right)$ and $\left(p_{1}, \ldots, p_{n}\right)$ be two non-negative $n$-tuples such that $\sum_{k=1}^{n} p_{k} x_{k} \geq$ $x_{i}>c$ for $i=1, \ldots, n, c \in[0, a]$ and $\sum_{k=1}^{n} p_{k} x_{k} \in[0, a]$. Also let $h:[0, a] \rightarrow \mathbb{R}$ be a positive function and $f:[0, a] \rightarrow \mathbb{R}$ be a function. If $\frac{f(x)}{h(x-c)}$ is increasing for $x>c$ on $[0, a]$, then

$$
\sum_{k=1}^{n} p_{k} f\left(x_{k}\right) \leq \frac{\sum_{k=1}^{n} p_{k} h\left(x_{k}-c\right)}{h\left(\sum_{k=1}^{n} p_{k} x_{k}-c\right)} f\left(\sum_{k=1}^{n} p_{k} x_{k}\right) .
$$

Proof. Since $\sum_{k=1}^{n} p_{k} x_{k} \geq x_{j}>c$ for all $j=1, \ldots, n$ and $\frac{f(x)}{h(x-c)}$ is increasing on $[0, a]$,

$$
\frac{f\left(\sum_{k=1}^{n} p_{k} x_{k}\right)}{h\left(\sum_{k=1}^{n} p_{k} x_{k}-c\right)} \geq \frac{f\left(x_{j}\right)}{h\left(x_{j}-c\right)},
$$

that is,

$$
h\left(\sum_{k=1}^{n} p_{k} x_{k}-c\right) f\left(x_{j}\right) \leq h\left(x_{j}-c\right) f\left(\sum_{k=1}^{n} p_{k} x_{k}\right) .
$$

Multiplying above inequality by $p_{j}$ and taking sum for $j=1, \ldots, n$, one has

$$
h\left(\sum_{k=1}^{n} p_{k} x_{k}-c\right) \sum_{j=1}^{n} p_{j} f\left(x_{j}\right) \leq \sum_{j=1}^{n} p_{j} h\left(x_{j}-c\right) f\left(\sum_{k=1}^{n} p_{k} x_{k}\right) .
$$

This is equivalent to the required result.

The following theorem consists of the result for generalized Petrović's inequality for $h$-convex functions.

Theorem 2.1. Let $\left(x_{1}, \ldots, x_{n}\right)$ be non-negative $n$-tuples and $\left(p_{1}, \ldots, p_{n}\right)$ be positive $n$-tuples such that

$$
\sum_{k=1}^{n} p_{k} x_{k} \in[0, a] \text { and } \sum_{k=1}^{n} p_{k} x_{k} \geq x_{j} \geq c \text { for } j=1, \ldots, n \text { and } c \in[0, a] \text {. }
$$

Also let $h:[0, a] \rightarrow \mathbb{R}^{+}$be a supermultiplicative function such that

$$
h(\alpha)+h(1-\alpha) \leq 1, \text { for all } \alpha \in(0,1)
$$

If $f:[0, a] \rightarrow \mathbb{R}$ be an $h$-convex function on $[0, a]$, then 


$$
\begin{aligned}
\sum_{j=1}^{n} p_{j} f\left(x_{j}\right) & \leq \frac{\sum_{j=1}^{n} p_{j} h\left(x_{j}-c\right)}{h\left(\sum_{k=1}^{n} p_{k} x_{k}-c\right)} f\left(\sum_{k=1}^{n} p_{k} x_{k}\right) \\
& +\left(\sum_{j=1}^{n} p_{j}-\frac{\sum_{j=1}^{n} p_{j} h\left(x_{j}-c\right)}{h\left(\sum_{k=1}^{n} p_{k} x_{k}-c\right)}\right) f(c) .
\end{aligned}
$$

Proof. Suppose $f$ is $h$-convex and

$$
P_{h}(x)=\frac{f(x)-f(c)}{h(x-c)} .
$$

We take $y>x>c$ and $x=\alpha y+(1-\alpha) c$, then

$$
P_{h}(x)=\frac{f(\alpha y+(1-\alpha) c)-f(c)}{h(\alpha y+(1-\alpha) c-c)} \leq \frac{h(\alpha) f(y)+[h(1-\alpha)-1] f(c)}{h(\alpha(y-c))} .
$$

Using the fact that $h$ is supermultiplicative, one has

$$
P_{h}(x) \leq \frac{h(\alpha) f(y)+[h(1-\alpha)-1] f(c)}{h(\alpha) h(y-c))}
$$

Since $h(1-\alpha)-1 \leq-h(\alpha)$, this implies

$$
P_{h}(x) \leq \frac{f(y)}{h(y-c)}-\frac{f(c)}{h(y-c)}=P_{h}(y)
$$

As we have proved if $f$ is $h$-convex, then $\frac{f(x)-f(c)}{h(x-c)}$ is increasing for $x>c$ so substituting $f(x)$ by $f(x)-f(c)$ in Lemma 2.1, one has

$$
\sum_{j=1}^{n} p_{j}\left(f\left(x_{j}\right)-f(c)\right) \leq \frac{\sum_{j=1}^{n} p_{j} h\left(x_{j}-c\right)}{h\left(\sum_{k=1}^{n} p_{k} x_{k}-c\right)}\left[f\left(\sum_{k=1}^{n} p_{k} x_{k}\right)-f(c)\right] .
$$

The above inequality leads to the required result.

The following theorem is a simple consequence of the above theorem just by taking $c=0$. It can be considered as Petrović's inequality for $h$-convex functions.

Theorem 2.2. Let the conditions given in Theorem 2.1 are valid. If $f:[0, a] \rightarrow \mathbb{R}$ be an $h$-convex function on $[0, a]$, then

$$
\sum_{j=1}^{n} p_{j} f\left(x_{j}\right) \leq \frac{\sum_{j=1}^{n} p_{j} h\left(x_{j}\right)}{h\left(\sum_{k=1}^{n} p_{k} x_{k}\right)} f\left(\sum_{k=1}^{n} p_{k} x_{k}\right)+\left(\sum_{j=1}^{n} p_{j}-\frac{\sum_{j=1}^{n} p_{j} h\left(x_{j}\right)}{h\left(\sum_{k=1}^{n} p_{k} x_{k}\right)}\right) f(0) .
$$

From Theorem 2.1, one can get a generalization of Petrović's inequality. 
Theorem 2.3. Suppose that $\left(x_{1}, \ldots, x_{n}\right)$ and $\left(p_{1}, \ldots, p_{n}\right)$ be two non-negative $n$-tuples such that $\sum_{k=1}^{n} p_{k} x_{k} \geq$ $x_{i}>c$ for $i=1, \ldots, n, c \in[0, a]$ and $\sum_{k=1}^{n} p_{k} x_{k} \in[0, a]$. If $f:[0, a] \rightarrow \mathbb{R}$ be a convex function on $[0, a]$, then

$$
\sum_{j=1}^{n} p_{j} f\left(x_{j}\right) \leq \frac{\sum_{j=1}^{n} p_{j}\left(x_{j}-c\right)}{\left(\sum_{k=1}^{n} p_{k} x_{k}-c\right)} f\left(\sum_{k=1}^{n} p_{k} x_{k}\right)+\left(\sum_{j=1}^{n} p_{j}-\frac{\sum_{j=1}^{n} p_{j}\left(x_{j}-c\right)}{\left(\sum_{k=1}^{n} p_{k} x_{k}-c\right)}\right) f(c)
$$

Proof. Let us consider $h(x)=x$, then clearly $h$ is supermultiplicative and condition (2.4) is valid. Taking this value of $h$ in Theorem 2.1 leads us to required result.

Remark 2.1. Taking $h(x)=x$ in Theorem 2.2 or $c=0$ in Theorem 2.3 leads to Theorem 1.1.

\section{Generalized Petrović's inequality for h-COncave function}

In the previous section, one can see that the condition on function $h$ given in (2.4) restrict us to give Petrović's type inequalities for particular cases of $h$-convex functions given in Remark 1.1. If we consider reverse inequality in (2.4), then it covers those particular cases but instead of $h$-convex function, we have $h$-concave function.

Lemma 3.1. Suppose that $\left(x_{1}, \ldots, x_{n}\right)$ and $\left(p_{1}, \ldots, p_{n}\right)$ be two non-negative $n$-tuples such that $\sum_{k=1}^{n} p_{k} x_{k} \geq x_{i}$ for $i=1, \ldots, n$ and $\sum_{k=1}^{n} p_{k} x_{k} \in[0, a]$. Also let $h:[0, a] \rightarrow \mathbb{R}$ be a positive function and $f:[0, a] \rightarrow \mathbb{R}$ be a function. If $\frac{f(x)}{h(x-c)}$ is decreasing for $x>c$ on $[0, a]$, then reverse of inequality (2.2) is valid.

Proof. The proof is similar to the Lemma 2.1 .

In the following theorem, reverse of (2.5) has been concluded. The notable thing is the requirements of submultiplicity and reverse of (2.4) for function $h$ along with $h$-concavity of the function $f$.

Theorem 3.1. Let $\left(x_{1}, \ldots, x_{n}\right)$ be non-negative $n$-tuples and $\left(p_{1}, \ldots, p_{n}\right)$ be positive $n$-tuples and the conditions given in (2.3) are valid. Also let $h:[0, a] \rightarrow \mathbb{R}^{+}$be a submultiplicative function such that

$$
h(\alpha)+h(1-\alpha) \geq 1, \text { for all } \alpha \in(0,1)
$$

If $f:[0, a] \rightarrow \mathbb{R}$ be an $h$-concave function on $[0, a]$, then reverse of $(2.5)$ is valied, that is,

$$
\begin{aligned}
\sum_{j=1}^{n} p_{j} f\left(x_{j}\right) \geq & \frac{\sum_{j=1}^{n} p_{j} h\left(x_{j}-c\right)}{h\left(\sum_{k=1}^{n} p_{k} x_{k}-c\right)} f\left(\sum_{k=1}^{n} p_{k} x_{k}\right) \\
& +\left(\sum_{j=1}^{n} p_{j}-\frac{\sum_{j=1}^{n} p_{j} h\left(x_{j}-c\right)}{h\left(\sum_{k=1}^{n} p_{k} x_{k}-c\right)}\right) f(c)
\end{aligned}
$$


Proof. First we will prove that $\frac{f(x)-f(c)}{h(x-c)}$ is decreasing for $x>c$ when $f$ is $h$-concave function. For this purpose consider

$$
P_{h}(x)=\frac{f(x)-f(c)}{h(x-c)} .
$$

We take $y>x>c$ and $x=\alpha y+(1-\alpha) c$, then

$$
P_{h}(x)=\frac{f(\alpha y+(1-\alpha) c)-f(c)}{h(\alpha y+(1-\alpha) c-c)} \geq \frac{h(\alpha) f(y)+[h(1-\alpha)-1] f(c)}{h(\alpha(y-c))} .
$$

Using the fact that $h$ is submultiplicative, so we have

$$
P_{h}(x) \geq \frac{h(\alpha) f(y)+[h(1-\alpha)-1] f(c)}{h(\alpha) h(y-c))}
$$

Since $h(1-\alpha)-1 \geq-h(\alpha)$, this implies

$$
P_{h}(x) \geq \frac{f(y)}{h(y-c)}-\frac{f(c)}{h(y-c)}=P_{h}(y) .
$$

This proves that $\frac{f(x)-f(c)}{h(x-c)}$ is decreasing in $[0, a]$ for $x>c$. Now substituting $f(x)$ by $f(x)-f(c)$ in Lemma 3.1 give us the required result.

In the following theorem, we give Petrović's inequality for $h$-concave functions. It is simple consequence of the previous theorem by just taking $c=0$.

Theorem 3.2. Let the conditions given in Theorem 3.1 are valid. If $f:[0, a] \rightarrow \mathbb{R}$ be an $h$-concave function on $[0, a]$, then the reverse of $(2.6)$ is valid.

In the following theorem, we give the generalized Petrović's inequality for concave functions.

Theorem 3.3. Suppose that $\left(x_{1}, \ldots, x_{n}\right)$ and $\left(p_{1}, \ldots, p_{n}\right)$ be two non-negative $n$-tuples such that $\sum_{k=1}^{n} p_{k} x_{k} \geq$ $x_{i}>c$ for $i=1, \ldots, n, c \in[0, a]$ and $\sum_{k=1}^{n} p_{k} x_{k} \in[0, a]$. If $f:[0, a] \rightarrow \mathbb{R}$ be concave function on $[0, a]$, then the reverse of $(2.7)$ is valid.

Proof. If we take $h(x)=x$ in (3.2), we get the required result.

Remark 3.1. By Taking $h(x)=x$ in Theorem 3.2 or $c=0$ in Theorem 3.3, one can get the reverse of inequality (1.2) in the case when $f$ is concave function.

In the following theorem, we give the Petrović's type inequality for reverse $P$-functions.

Theorem 3.4. Suppose that $\left(x_{1}, \ldots, x_{n}\right)$ and $\left(p_{1}, \ldots, p_{n}\right)$ be two non-negative $n$-tuples such that $\sum_{k=1}^{n} p_{k} x_{k} \geq$ $x_{i}$ for $i=1, \ldots, n$ and $\sum_{k=1}^{n} p_{k} x_{k} \in[0, a]$. If $f:[0, a] \rightarrow \mathbb{R}$ be a reverse $P$-function on $[0, a]$, then

$$
\sum_{j=1}^{n} p_{j} f\left(x_{j}\right) \geq \sum_{j=1}^{n} p_{j} f\left(\sum_{k=1}^{n} p_{k} x_{k}\right)
$$


Proof. If we take $h(x)=1$, then it fulfils the condition of Theorem 3.1 and follows the required result.

In the following theorem, we give the generalized Petrović's type inequality for reverse Godunova-Levin functions.

Theorem 3.5. Suppose that $\left(x_{1}, \ldots, x_{n}\right)$ and $\left(p_{1}, \ldots, p_{n}\right)$ be two non-negative $n$-tuples such that $\sum_{k=1}^{n} p_{k} x_{k} \geq$ $x_{i}>c$ for $i=1, \ldots, n, c \in[0, a]$ and $\sum_{k=1}^{n} p_{k} x_{k} \in[0, a]$. If $f:[0, a] \rightarrow \mathbb{R}$ be a reverse Godunova-Levin function on $[0, a]$, then

$$
\begin{aligned}
\sum_{j=1}^{n} p_{j} f\left(x_{j}\right) & \geq\left(\sum_{k=1}^{n} p_{k} x_{k}-c\right) \sum_{j=1}^{n} \frac{p_{j}}{x_{j}-c} f\left(\sum_{k=1}^{n} p_{k} x_{k}\right) \\
& +\left(\sum_{j=1}^{n} p_{j}-\left(\sum_{k=1}^{n} p_{k} x_{k}-c\right) \sum_{j=1}^{n} \frac{p_{j}}{x_{j}-c}\right) f(c) .
\end{aligned}
$$

Proof. Consider $h(x)=\frac{1}{x}$, then

$$
h(\alpha)+h(1-\alpha)=\frac{1}{\alpha}+\frac{1}{1-\alpha}>1 \text { for all } \alpha \in(0,1) .
$$

Using above value of $h$ in Theorem 3.1 gives the required result.

The following theorem is a simple consequence of the previous theorem. It is worth stating as Petrović's type inequality for reverse Godunova-Levin functions.

Theorem 3.6. Let the conditions given in Theorem 3.1 are valid. If $f:[0, a] \rightarrow \mathbb{R}$ be a reverse GodunovaLevin function on $[0, a]$, then

$$
\begin{aligned}
\sum_{j=1}^{n} p_{j} f\left(x_{j}\right) & \geq\left(\sum_{k=1}^{n} p_{k} x_{k}\right) \sum_{j=1}^{n} \frac{p_{j}}{x_{j}} f\left(\sum_{k=1}^{n} p_{k} x_{k}\right) \\
& +\left(\sum_{j=1}^{n} p_{j}-\left(\sum_{k=1}^{n} p_{k} x_{k}\right) \sum_{j=1}^{n} \frac{p_{j}}{x_{j}}\right) f(0) .
\end{aligned}
$$

Proof. Putting $c=0$ in Theorem 3.6 leads to required result.

Before giving two important theorems, let us consider

$$
H(h)=h(\alpha)+h(1-\alpha)-1, \alpha \in(0,1)
$$

then for different values of $h$, that is, for $\alpha^{s}$ and $\frac{1}{\alpha^{s}}$, we take

$$
g_{1}(\alpha):=H\left(\alpha^{s}\right)=\alpha^{s}+(1-\alpha)^{s}-1
$$

and

$$
g_{2}(\alpha):=H\left(\frac{1}{\alpha^{s}}\right)=\frac{1}{\alpha^{s}}+\frac{1}{(1-\alpha)^{s}}-1, \text { where } s \in(0,1) .
$$




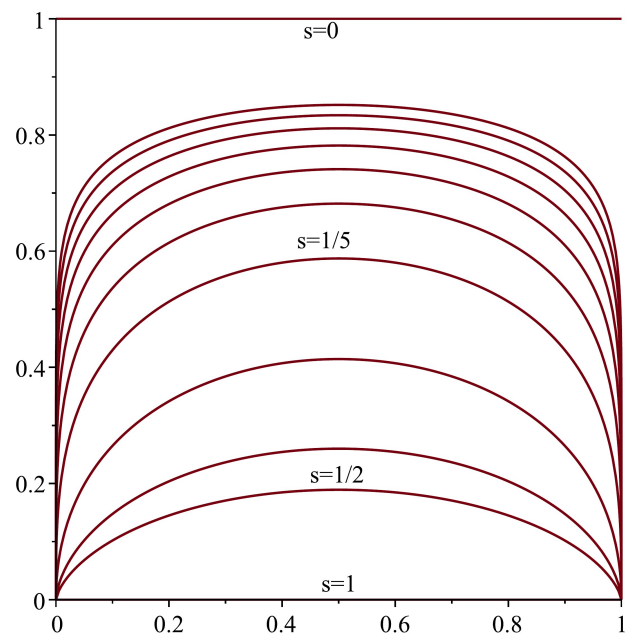

Figure 1. Graph of $g_{1}$ at different value of $s$. One can see that $g_{1}$ is positive for $\alpha \in(0,1)$ and at different value of $s$. The line at bottom is at $s=1$, the next curve is for $s=\frac{1}{2}$ and so on.

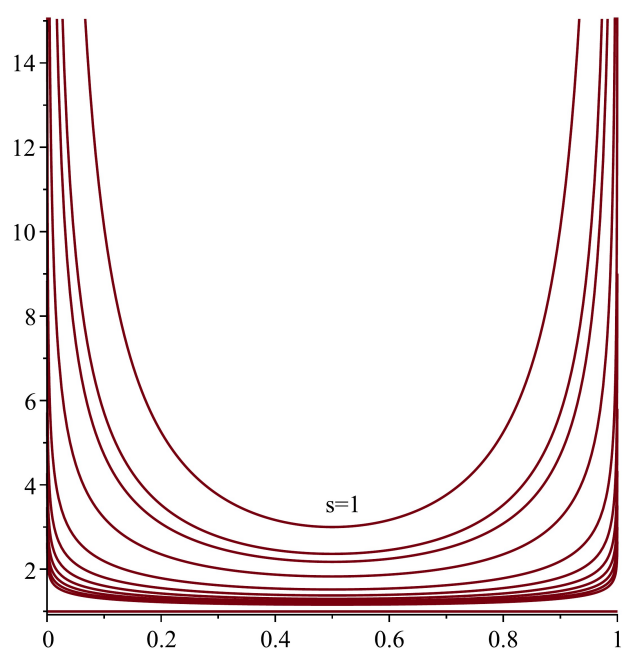

Figure 2. Graph of $g_{2}$ at different value of $s$. One can see that $g_{2}$ is positive for $\alpha \in(0,1)$ and at different value of $s$. The curve at top is at $s=1$, the below one is for $s=\frac{1}{2}$ and so on.

From Figures 1 and 2, one can see that $g_{1}$ and $g_{2}$ are positive, therefore $h(\alpha)=\alpha^{s}$ and $h(\alpha)=\frac{1}{\alpha^{s}}$ for $\alpha, s \in(0,1)$ satisfied the conditions of Theorem 3.1, but these functions does not satisfy the conditions of Theorem 2.1. Hence the above two particular values of $h$ in Theorem 3.1 leads us the following two theorems.

Theorem 3.7. Suppose that $\left(x_{1}, \ldots, x_{n}\right)$ and $\left(p_{1}, \ldots, p_{n}\right)$ be two non-negative $n$-tuples such that $\sum_{k=1}^{n} p_{k} x_{k} \geq$ $x_{i}>c$ for $i=1, \ldots, n, c \in[0, a]$ and $\sum_{k=1}^{n} p_{k} x_{k} \in[0, a]$. If $f:[0, a] \rightarrow \mathbb{R}$ be a reverse s-Godunova-Levin 
function on $[0, a]$. Then

$$
\begin{aligned}
\sum_{j=1}^{n} p_{j} f\left(x_{j}\right) & \geq\left(\sum_{k=1}^{n} p_{k} x_{k}-c\right)^{s} \sum_{j=1}^{n} \frac{p_{j}}{\left(x_{j}-c\right)^{s}} f\left(\sum_{k=1}^{n} p_{k} x_{k}\right) \\
& +\left(\sum_{j=1}^{n} p_{j}-\left(\sum_{k=1}^{n} p_{k} x_{k}-c\right)^{s} \sum_{j=1}^{n} \frac{p_{j}}{\left(x_{j}-c\right)^{s}}\right) f(c) .
\end{aligned}
$$

Theorem 3.8. Suppose that $\left(x_{1}, \ldots, x_{n}\right)$ and $\left(p_{1}, \ldots, p_{n}\right)$ be two non-negative $n$-tuples such that $\sum_{k=1}^{n} p_{k} x_{k} \geq$ $x_{i}>c$ for $i=1, \ldots, n, c \in[0, a]$ and $\sum_{k=1}^{n} p_{k} x_{k} \in[0, a]$. If $f:[0, a] \rightarrow \mathbb{R}$ be a s-concave function on $[0, a]$, then

$$
\begin{aligned}
\sum_{j=1}^{n} p_{j} f\left(x_{j}\right) & \geq\left(\sum_{k=1}^{n} p_{k} x_{k}-c\right)^{s} \sum_{j=1}^{n} \frac{p_{j}}{\left(x_{j}-c\right)^{s}} f\left(\sum_{k=1}^{n} p_{k} x_{k}\right) \\
& +\left(\sum_{j=1}^{n} p_{j}-\left(\sum_{k=1}^{n} p_{k} x_{k}-c\right) \sum_{j=1}^{n} \frac{p_{j}}{\left(x_{j}-c\right)^{s}}\right) f(c) .
\end{aligned}
$$

If we take $c=0$, then we have following Petrović's type inequalities.

Theorem 3.9. Let the conditions given in Theorem 1.1 are valid. If $f:[0, a] \rightarrow \mathbb{R}$ be a reverse s-GodunovaLevin function on $[0, a]$, then

$$
\begin{aligned}
\sum_{j=1}^{n} p_{j} f\left(x_{j}\right) & \geq\left(\sum_{k=1}^{n} p_{k} x_{k}\right)^{s} \sum_{j=1}^{n} \frac{p_{j}}{\left(x_{j}\right)^{s}} f\left(\sum_{k=1}^{n} p_{k} x_{k}\right) \\
& +\left(\sum_{j=1}^{n} p_{j}-\left(\sum_{k=1}^{n} p_{k} x_{k}\right)^{s} \sum_{j=1}^{n} \frac{p_{j}}{\left(x_{j}\right)^{s}}\right) f(c) .
\end{aligned}
$$

Theorem 3.10. Let the conditions given in Theorem 1.1 are valid. If $f:[0, a] \rightarrow \mathbb{R}$ be a s-concave function on $[0, a]$, then

$$
\begin{aligned}
\sum_{j=1}^{n} p_{j} f\left(x_{j}\right) & \geq\left(\sum_{k=1}^{n} p_{k} x_{k}\right)^{s} \sum_{j=1}^{n} \frac{p_{j}}{\left(x_{j}\right)^{s}} f\left(\sum_{k=1}^{n} p_{k} x_{k}\right) \\
& +\left(\sum_{j=1}^{n} p_{j}-\left(\sum_{k=1}^{n} p_{k} x_{k}\right)^{s} \sum_{j=1}^{n} \frac{p_{j}}{\left(x_{j}\right)^{s}}\right) f(0) .
\end{aligned}
$$

Proof. Put $c=0$ in Theorem 3.8, one has the required result.

\section{Concluding Remarks}

This paper generalized the Petrović's inequality for $h$-convex ( $h$-concave) functions. It has been noted that under certain conditions on $h$, Theorem 2.1 provide the generalization of Petrović's inequality for $h$-convex functions, but this generally does not leads to Godunova-Levin functions, $P$-functions, $s$-GodunovaLevin functions and $s$-convex functions. Theorem 3.1 give the Petrović's inequality for $h$-concave under certain condition on $h$. Interesting, it give reverse of those particular cases for which Theorem 2.1 fails. 
It is an open problem to find such generalization of Petrović's inequality for $h$-convex functions with some suitable conditions on $h$, which lead to all particular cases of $h$-convex functions specially mentioned in Remark 1.1.

\section{ACKNOWLEDGEMENT}

The authors are very grateful to the editor and reviewers for their careful and meticulous reading of the paper. The research work of the 1st author is supported by Higher Education Commission of Pakistan under NRPU 2016, Project No. 5421.

\section{REFERENCES}

[1] S. Varošanec, On h-Convexity, J. Math. Anal. Appl. 326(1) (2007), 303-311.

[2] S. S. Dragomir, J. E. Pečarić and L. E. Persson , Some Inequalities of Hadamard inequality, Soochow J. Math. 21(3) (1995), 335-341.

[3] A. Házy, Bernstein-Doetsch Type Results for $h$-Convex Functions, Math. Inequal. Appl. 14(3) (2011), $499-508$.

[4] S. S Dragomir, On Hadamards Inequality for Convex Functions on the Co-ordinates in a Rectangle from the Plane, Taiwanese J. Math. 5(4) (2001), 775-788.

[5] M. Alomari, M. A. Latif, On Hadmard-Type Inequalities for $h$-Convex Functions on the Co-ordinates, Int. J. Math. Anal. 3(33) (2009), 1645-1656.

[6] M. Petrović's, Sur Une Fonctionnelle, Publ. Math. Univ. Belgrade 1 (1932), 146-149.

[7] J. E. Pečarić, F. Proschan and Y. L. Tong, Convex Functions, Partial Orderings and Statistical Applications, Academic Press, New York, 1991.

[8] M. Bombardelli, S. Varoanec, Properties of $h$-Convex Functions Related to the HermiteHadamardFejr Inequalities, Comput. Math. Appl. 58(9) (2009), 1869-1877.

[9] A. Olbryś, On Separation by h-Convex Function, Tatra Mt. Math. Publ. 62(1) (2015), 105-111.

[10] S. Butt, J. Pečarić and A. U. Rehman, Exponential Convexity of Petrović and Related Functional, J. Inequal. Appl. 2011(1) (2011), 16 pp.

[11] J. Pečarić and V. Čuljak, Inequality of Petrović and Giaccardi for Convex Function of Higher Order, Southeast Asian Bull. Math. 26(1) (2003), 57-61.

[12] J. E. Pečarić, On the Petrović Inequality for Convex Functions, Glasnik Matematicki 18(38) (1983), 77-85.

[13] M. K. Bakula, J. Pečarić and M. Ribičić, Companion Inequalities to Jensen's Inequality for $m$-Convex and ( $\alpha, m)$-Convex Functions, J. Inequal. Pure Appl. Math. 7(5) (2006), 32 pp.

[14] A. U. Rehman, M. Mudessir, H. T. Fazal and G. Farid, Petrović's Inequality on Coordinates and Related Results, Cogent Math. 3(1) (2016), 11 pp.

[15] J. Pečarić and J. Peric, Improvements of the Giaccardi and the Petrovic Inequality and Related Stolarsky Type Means, An. Univ. Craiova, Ser. Mat. Inf. 39(1) (2012), 65-75. 